\title{
High-beta plasma effects in a low-pressure helicon plasma
}

\author{
C. S. Corr and R. W. Boswell \\ Space Plasma, Power and Propulsion Group (SP3), Research School of Physical Science and Engineering, \\ Australian National University, Canberra ACT 0200, Australia
}

(Received 25 July 2007; accepted 2 October 2007; published online 7 December 2007)

\begin{abstract}
In this work, high-beta plasma effects are investigated in a low-pressure helicon plasma source attached to a large volume diffusion chamber. When operating above an input power of $900 \mathrm{~W}$ and a magnetic field of $30 \mathrm{G}$ a narrow column of bright blue light (due to Ar II radiation) is observed along the axis of the diffusion chamber. With this blue mode, the plasma density is axially very uniform in the diffusion chamber; however, the radial profiles are not, suggesting that a large diamagnetic current might be induced. The diamagnetic behavior of the plasma has been investigated by measuring the temporal evolution of the magnetic field $\left(B_{z}\right)$ and the plasma kinetic pressure when operating in a pulsed discharge mode. It is found that although the electron pressure can exceed the magnetic field pressure by a factor of 2 , a complete expulsion of the magnetic field from the plasma interior is not observed. In fact, under our operating conditions with magnetized ions, the maximum diamagnetism observed is $\sim 2 \%$. It is observed that the magnetic field displays the strongest change at the plasma centre, which corresponds to the maximum in the plasma kinetic pressure. These results suggest that the magnetic field diffuses into the plasma sufficiently quickly that on a long time scale only a slight perturbation of the magnetic field is ever observed. (C) 2007 American Institute of Physics. [DOI: 10.1063/1.2802080]
\end{abstract}

\section{INTRODUCTION}

The interaction of plasmas with magnetic fields is of fundamental importance to understanding space and laboratory plasma physics phenomena, such as the impact of the solar wind on the earth's magnetic field, particle diffusion processes across magnetic field, and spacecraft propulsion. ${ }^{1-4}$ In particular, it is generally thought that these dense plasmas exhibit a significant diamagnetism, which changes the magnetic field inside the plasma. An important parameter in determining this effect is the plasma "beta,", which is the ratio of the particle pressure to the external magnetic field pressure, and is given by

$$
\beta=\frac{n k T}{B^{2} / 2 \mu_{0}},
$$

where $n$ and $T$ denote the plasma density and temperature, respectively, and $B$ is the ambient magnetic field. Studies of high-beta plasma in space have been performed in the field of the Earth's magnetosphere as one of the scenario's of auroral substorms. ${ }^{6,7}$ At the inner edge of the plasma sheet the plasma density is high, and the magnetic lines of force are in closed form. A configuration of high-beta plasma contained in a curved magnetic field is realized here. Coronal loops consist of a high-beta plasma or fast plasma flow surrounded by the low-beta corona. Simulation of space in a laboratory is of great scientific interest to understanding these naturally occurring space phenomena.

The conventional picture for the situation of high-beta plasmas is that the external magnetic field will be excluded from the interior of the plasma and essentially the plasma will propagate downstream by pushing or "ploughing" the field aside. However, the magnetic field penetration rates can greatly exceed the plasma diffusion time, leading to a more complex picture. Experiments on the propagation of neutralized ion beams transverse to a magnetic field have shown the existence of fast penetration of the magnetic field into the beam. ${ }^{8}$ It has been found that although the beam beta can be as high as 400 , the largest value of diamagnetism was found to be $10 \%$ to $15 \%$. The authors concluded that in this highbeta limit the magnetic field diffuses rapidly into the propagating ion beam, on a time scale much shorter than the beam rise time and that beam propagation does not occur by diamagnetic exclusion of the transverse magnetic field. Essentially, the magnetic field is present inside the beam at all times. Recent rocket-borne experiments have also reported fast diffusion of the magnetic field for a high-density, heavy ion beam propagating nearly perpendicular to the Earth's magnetic field in the presence of the ambient space plasma. ${ }^{9}$

There has been relatively little in the way of publications on high-beta plasmas and measurement of the plasma diamagnetism. Recently, Stenzel and Urrutia ${ }^{10}$ investigated the interaction of a dense discharge plasma with a weak magnetic field of $5 \mathrm{G}$; i.e., the ions are not magnetized. They found that when the electron pressure exceeded the magnetic field pressure with a $\beta=5$, a magnetic hole is formed in the plasma interior. However, in their measurements, the plasma diamagnetism was found to be $\sim 15 \%$ with a beta equal to 1 . They did not investigate magnetic fields of higher field strength where the ions are magnetized and have Larmor radii less than the system dimensions.

In the present work, we investigate high-beta phenomena in a plasma system consisting of a helicon source attached to a much larger diffusion chamber. Helicon waves are bounded electromagnetic waves in magnetized plasma with frequencies between the ion cyclotron frequency and the electron cyclotron frequency. ${ }^{11-13}$ They are of considerable interest 


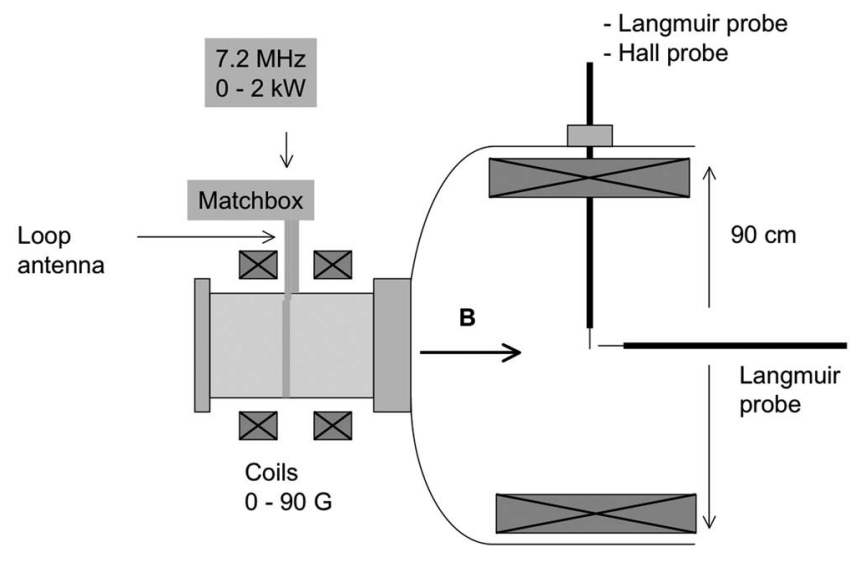

FIG. 1. A schematic of WOMBAT and the diagnostic setup.

because they can propagate at much higher densities than many other waves in plasmas (the name "helicon waves" comes from studies of these waves in solids). Helicon waves provide an opportunity to reach very high densities in electrodeless discharges and can have very high fractional ionization. There is a growing list of applications, which includes radio-frequency (rf) ion lasers of large radii. ${ }^{14}$ Although helicon sources have been around for some time, they are not yet well understood.

In this paper, we investigate a high-beta helicon plasma with magnetized ions. In particular we explore the plasma beta and the diamagnetic behavior. The paper is organized as follows. The experimental setup and diagnostic methods are described in Sec. II. The basic plasma properties and the "blue" core results are presented in Sec. III. Section IV details the high-beta effects and the diamagnetic properties of the plasma. Some conclusions are drawn in Sec. V.

\section{EXPERIMENTAL ARRANGEMENT AND DIAGNOSTICS}

The large plasma diffusion system WOMBAT (Waves On Magnetized Beams And Turbulence) consists of a glass source tube $50 \mathrm{~cm}$ long and $18 \mathrm{~cm}$ in diameter attached on axis to a large stainless steel diffusion chamber $200 \mathrm{~cm}$ long and $90 \mathrm{~cm}$ inner diameter, as shown in Fig. 1. A glass plate terminates the other end of the source. A steady axial magnetic field is maintained by a set of external solenoids surrounding the source and a large solenoid inside the diffusion chamber. In these experiments, the source solenoids were not used. As a result the magnetic field was highly uniform inside the diffusion chamber but divergent in the source. The maximum achievable magnetic field in the diffusion chamber is $200 \mathrm{G}$. In this work, we focus on two magnetic field cases: 34 and $185 \mathrm{G}$.

A base pressure of $4 \times 10^{-6}$ Torr is maintained in the vacuum chamber by a turbomolecular pump attached to the diffusion chamber end opposite the source. Operating pressures of 0.1-3 mTorr are set using a mass flow controller and an ion gauge. A rf power supply provides up to $2 \mathrm{~kW}$ of forward power at a frequency of $7.2 \mathrm{MHz}$ to one end of a single loop antenna via a matching network, while the other end is grounded. The antenna is $20 \mathrm{~cm}$ in diameter, $1 \mathrm{~cm}$ wide, and $0.3 \mathrm{~cm}$ thick, and is positioned around the outside of the glass source tube, directly below the matching box. It is located $30 \mathrm{~cm}$ from the source/diffusion chamber interface. For these experiments, the plasma was operated with an ON time of $8 \mathrm{~ms}$ and OFF time of $60 \mathrm{~ms}$ so as to reduce burn-out of internal diagnostics.

A noncompensated, translating Langmuir probe was inserted radially into the diffusion chamber $50 \mathrm{~cm}$ from the source/diffusion chamber interface (Fig. 1). The probe consisted of a $0.2 \mathrm{~mm}$ diameter and $8 \mathrm{~mm}$ long nickel wire, shielded by a $6 \mathrm{~mm}$ diameter grounded steel tubing extending the length of the probe up to the probe tip. The radial translation of the probe was set using a computer controlled stepper motor arrangement that allowed the probe tip position to be selected with an accuracy of a few micrometers.

The plasma density $(n)$, electron temperature $\left(T_{e}\right)$, plasma potential $\left(V_{p}\right)$, and floating potential $\left(V_{f}\right)$ were determined from the $I(V)$ characteristics of the cylindrical probe: (i) $V_{p}$ from the zero of the second derivative of the $I(V)$ traces; (ii) $V_{f}$ from the zero crossing of the $I(V)$ curve; (iii) $n$ from the ion saturation current, $i_{\text {sat }}=0.6 A n e u_{\mathrm{B}}$, where $u_{\mathrm{B}}$ is the Bohm velocity, $A$ the probe area, $n$ the plasma density, and $e$ the electron charge; and (iv) $T_{e}$ from a linear fit of the logarithm of the electron current assuming a Maxwellian electron distribution function. The Langmuir probe was operated using a LABVIEW program, and the bias voltage on the probe was swept between -50 and $+50 \mathrm{~V}$ to determine the plasma $I(V)$ characteristics.

The diamagnetic behavior of the plasma was studied by measuring the time-varying magnetic field using diamagnetic coils located inside and outside the diffusion chamber. A Hall probe was used to measure the temporal and spatial variation of the magnetic field through the discharge pulse. The Hall probe has three mutually perpendicular elements to measure the $X, Y$, and $Z$ components of the probe. A B-dot probe was used to investigate time varying magnetic fields occurring in the diffusion chamber. The probe is a $70 \mathrm{~cm}$ long tube and $0.5 \mathrm{~cm}$ in diameter with a five-turn winding of thin insulated copper wire at one end. The probe consists of two loops, one of which with its normal pointing in the axial direction to measure the axial component of $B$ and the other points perpendicular to this direction to measure the perpendicular component of $B$.

\section{BASIC PLASMA PROPERTIES AND THE "BLUE CORE"}

\section{A. Input power and magnetic field}

The temporal variation of the plasma density is shown in Fig. 2 through one time period of the plasma discharge. The operating conditions are an input power of $1708 \mathrm{~W}$ and gas pressure of $1 \mathrm{mTorr}$. The rise time of the plasma is very different depending on the magnetic field value used. As can be seen from Fig. 2, the rise time for the $185 \mathrm{G}$ case is much faster than that of the $34 \mathrm{G}$ case due to the increase in the axial diffusion. It is observed that the plasma density is greater by a factor of 2 for the higher magnetic field case.

The plasma density, measured at the radial center of the diffusion chamber, $50 \mathrm{~cm}$ from the source, as a function of 


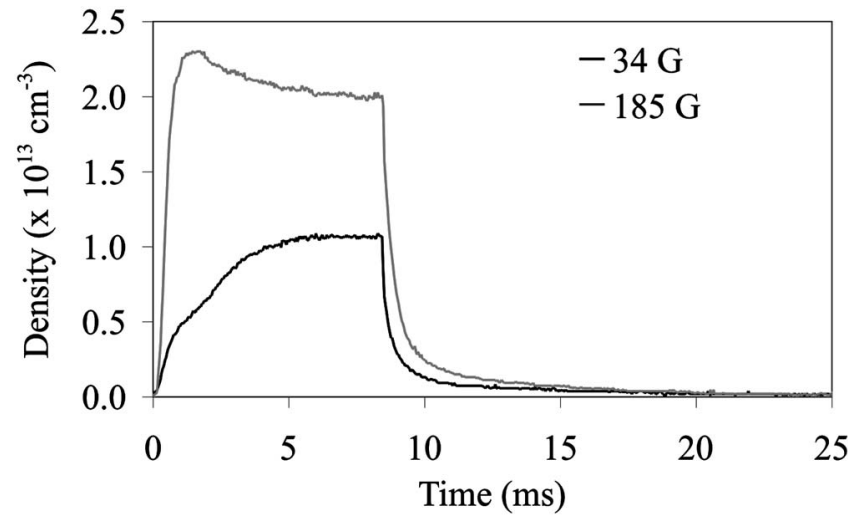

FIG. 2. The positive ion density measured through the discharge pulse.

input power and magnetic field strength, are shown in Figs. 3 and 4 , respectively. As the input power is increased the plasma density also increases, from $4 \times 10^{12} \mathrm{~cm}^{-3}$ at $\sim 600 \mathrm{~W}$ to $\sim 2 \times 10^{13} \mathrm{~cm}^{-3}$ at $\sim 1700 \mathrm{~W}$. There appears to be discontinuity in the increase, indicating a mode change occurring at $\sim 1100 \mathrm{~W}$. Similarly, as the magnetic field is increased the plasma density also increases (Fig. 4). No mode jump was found to occur when changing the magnetic field.

When operating above an input power of $900 \mathrm{~W}$ and a magnetic field of $30 \mathrm{G}$, a narrow column of bright blue light (due to Ar II radiation) is observed along the axis of the diffusion chamber. With this blue mode, the plasma density is axially very uniform; however, as discussed below, the radial profiles are not. It should be noted that a lowfrequency instability is observed when operating with magnetic fields above $100 \mathrm{G}$. The unstable behavior is localized at radial positions for $r>4 \mathrm{~cm}$ (i.e., outside the blue core) with the relative fluctuation amplitude increasing with increased radial position. This will be the presented in another publication.

For our situation, we consider a magnetic field moving into a highly collisional stationary plasma such that the electrons $\mathbf{E} \times \mathbf{B}$ velocity component can be ignored $\left(\nu_{e} \times \mathbf{B}=0\right)$ and Ohm's law becomes

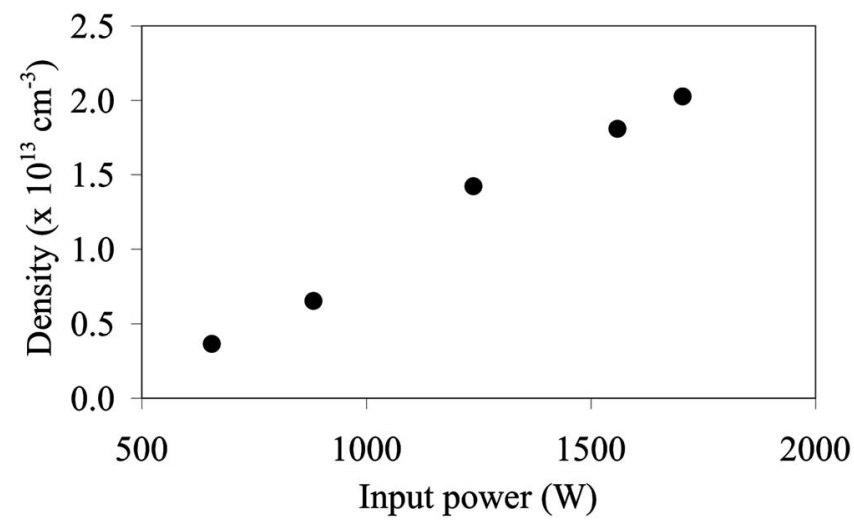

FIG. 3. The dependence of the plasma density with applied rf power.

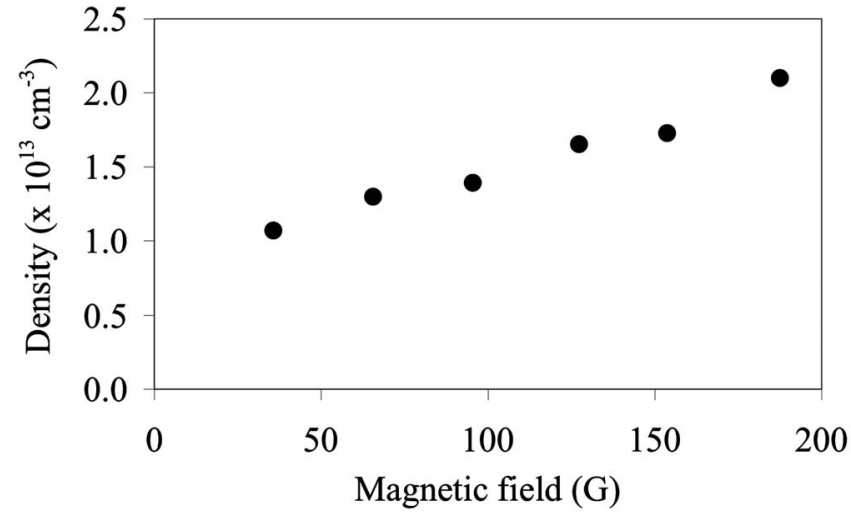

FIG. 4. The dependence of the plasma density with applied magnetic field.

$\mathbf{E}=\eta \mathbf{J}$,

where $\eta$ is the plasma resistivity and $J$ is the current density. For a highly ionized plasma, the resistivity is due to Coulomb collisions (Spitzer) and is independent of the density since the collision frequency increases proportional to $n_{e}$. Equation (1) expresses that the magnetic field is not "frozen" to the plasma fluid.

Substituting Maxwell's equation $\left(\boldsymbol{\nabla} \times \mathbf{B}=\mu_{0} j\right)$ into Faraday's law, one therefore obtains

$$
\begin{aligned}
\frac{\partial \mathbf{B}}{\partial t}=-\boldsymbol{\nabla} \times \mathbf{E} & =-\frac{\eta}{\mu_{0}} \boldsymbol{\nabla} \times \boldsymbol{\nabla} \times \mathbf{B} \\
& =-\frac{\eta}{\mu_{0}}\left[\nabla(\nabla \cdot B)-\nabla^{2} B\right] .
\end{aligned}
$$

Since $\boldsymbol{\nabla} \cdot B=0$ from Maxwell, we now obtain

$$
\frac{\partial \mathbf{B}}{\partial t}=\frac{\eta}{\mu_{0}} \nabla^{2} \mathbf{B} .
$$

The spatial variation of $B$ can be deduced from $\nabla^{2} \mathbf{B}=B / L^{2}$, giving

$$
\frac{\partial \mathbf{B}}{B \partial t}=\frac{\eta}{\mu_{0} L^{2}} .
$$

Integrating Eq. (4), one obtains

$$
\ln B=\frac{\eta t}{\mu_{0} L^{2}},
$$

a solution of which is

$$
B=B_{0} \exp (t / \tau) .
$$

Here, the characteristic time for magnetic field penetration into a plasma is given by

$$
\tau=\frac{\mu_{0} L^{2}}{\eta}
$$

where $\eta$ is the collisional plasma resistivity, $\mu_{0}$ is the permeability of free space, and $L$ is the characteristic scale length of the spatial variation of the magnetic field. The collisional plasma resistivity is dependent only on the electron temperature. For our situation, the magnetic field diffusion time is 


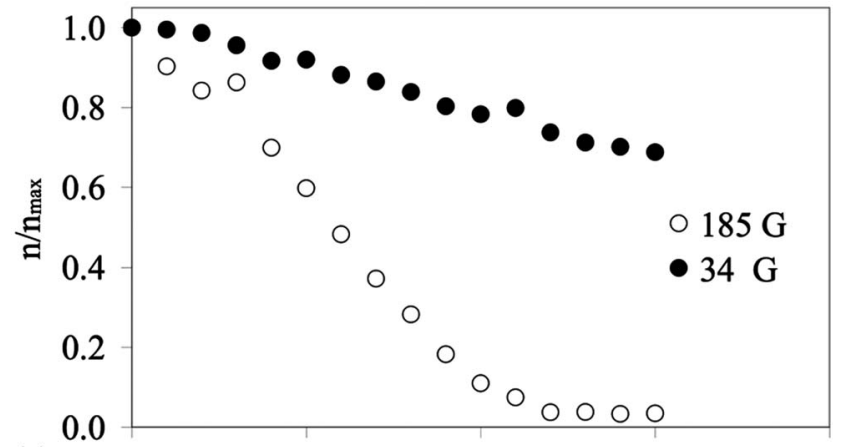

(a)

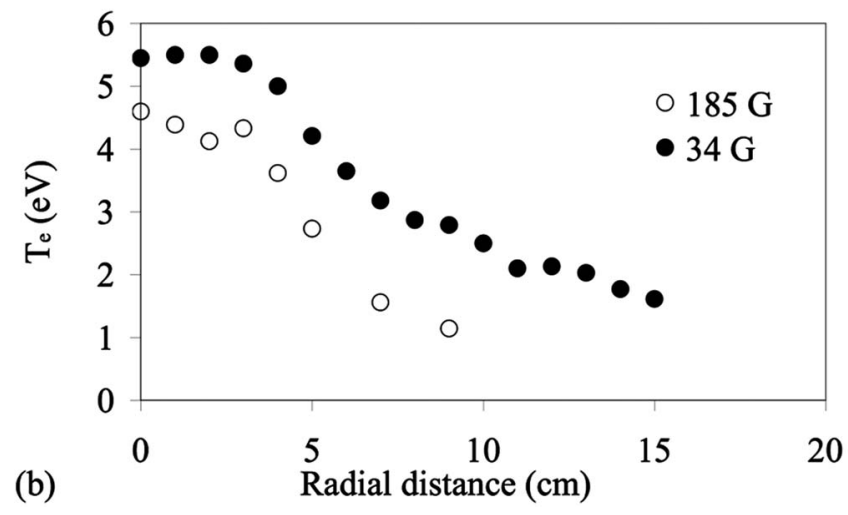

FIG. 5. The radial profile of (a) the normalized plasma density and (b) the electron temperature for an input power of $1708 \mathrm{~W}$ and gas pressure of 0.6 mTorr. It should be noted that a low-frequency instability is observed when operating with magnetic fields above $100 \mathrm{G}$ for $r>4 \mathrm{~cm}$ (i.e., outside the blue core). This will be the subject of a future publication.

approximately $50 \mu \mathrm{s}$, which is fast compared with the plasma rise time $(>500 \mu \mathrm{s})$.

\section{B. Radial dependence of plasma parameters}

The plasma parameters differ vastly depending on the magnetic field strength employed. In particular the radial profiles of the plasma density and the electron temperature are greatly modified. Figure 5 shows the radial variation of the normalized plasma density and electron temperature for an input power of $1708 \mathrm{~W}$ and magnetic fields of 185 and $34 \mathrm{G}$. The density is strongly modified at the higher magnetic field strength, showing a two-peak radial structure: one at $r=0 \mathrm{~cm}$ and another at $r=3 \mathrm{~cm}$. It is observed that the electron density and the electron temperature decrease as the plasma diffuses across the magnetic field. The density for both magnetic field cases are of similar values; i.e., $\sim 1.1$ $\times 10^{13} \mathrm{~cm}^{-3}$ for $34 \mathrm{G}$ and $\sim 2.1 \times 10^{13} \mathrm{~cm}^{-3}$ for $185 \mathrm{G}$. Cross-field diffusion is strongly modified with an increasing magnetic field, where the plasma density increases in the plasma center and decreases at the plasma edge $(r=15 \mathrm{~cm})$ with increasing field. The decrease in the density at $r=15 \mathrm{~cm}$ is a result of a decrease in the radial plasma diffusion. This shows that for the higher magnetic field strength there is more plasma confinement and axial diffusion is dominant. The full width at half-maximum (FWHM) of the radial density profiles is $\sim 15 \mathrm{~cm}$ for $34 \mathrm{G}$, while for $185 \mathrm{G}$ it is $\sim 10 \mathrm{~cm}$. For a magnetic field of $185 \mathrm{G}$, a distinct peak is observed to occur in the density at the plasma center for powers above $1000 \mathrm{~W}$. Furthermore, at $r \sim 3 \mathrm{~cm}$, there is a second peak in the density. This peaked plasma profile has previously been observed in WOMBAT and is a result of helicon wave propagation where the electric field maximum is located in the center and in addition, there is strong electron heating at the blue core edge due to the strong electrostatic confinement of the electrons. ${ }^{15}$

The radial profile of the electron temperature is shown in Fig. 5(b). For both magnetic fields, the temperature is highest at the discharge center $(5.5$ and $4.5 \mathrm{eV}$ for 34 and $185 \mathrm{G}$, respectively) and decays towards the radial edge. It is interesting to note that while the density does not vary strongly across the radius with a magnetic field of $34 \mathrm{G}$, the electron temperature does, decreasing from $5.5 \mathrm{eV}$ at the center to $\sim 1.5 \mathrm{eV}$ at $r=15 \mathrm{~cm}$.

The density gradient for the $185 \mathrm{G}$ case displays a maximum at $r \sim 4 \mathrm{~cm}$, whereas the $34 \mathrm{G}$ case remains fairly constant over the discharge radius. For both cases there is a maximum in the electron temperature gradient at $\sim 4 \mathrm{~cm}$.

\section{HIGH-BETA EFFECTS}

\section{A. Input power and magnetic field dependence}

The diamagnetic behavior of the plasma was investigated by measuring the temporal evolution of the magnetic field $\left(B_{z}\right)$ during the discharge pulse. For our operating conditions and with an applied field of $34 \mathrm{G}$, the above plasma parameters indicate that the peak electron pressure, i.e., $n k T_{e}=9.5 \times 10^{-6} \mathrm{~J} / \mathrm{cm}^{3}$, greatly exceeds the magnetic field pressure, i.e., $B_{0}^{2} / 2 \mu_{0} \sim 4.4 \times 10^{-6} \mathrm{~J} / \mathrm{cm}^{3}$, resulting in a beta of $\sim 2$. Therefore, one would expect to see a decrease in the applied magnetic field in the presence of the plasma.

The plasma diamagnetism was investigated using a diamagnetic loop situated internally on the axis of the blue core and also with a radially moveable Hall probe that measured the absolute value of the local magnetic field. An example the diamagnetic signal from the loop is shown in Fig. 6. Figure 6(a) shows the signal obtained from the diamagnetic loop and Fig. 6(b) shows the integration of this signal. Figure 6(b) indicates that there is a reduction in the magnetic field when the plasma is on. This is confirmed by direct measurement of the magnetic field using a Hall probe.

As can be seen in Fig. 7, the decrease of the local magnetic field strength is related to an increase in the applied input power and hence to the plasma kinetic pressure. For an input power of $656 \mathrm{~W}$, a very small reduction in the magnetic field of $\sim 0.4 \%$ is observed at the plasma center. Under these operating conditions, the plasma is referred to as a low-beta plasma. However, when the input power is increased, the magnetic field is observed to display a greater decrease, corresponding to a higher plasma electron pressure. These observations are in good agreement with the temporal variation of the plasma density, as shown in Fig. 2. For the case shown in Fig. 7, a magnetic field of $34 \mathrm{G}$ decreases by nearly $2 \%$ when an input power of $1708 \mathrm{~W}$ is applied. The trend of the diamagnetic measurements for various plasma beta, is shown in Fig. 8. For these measurements, the magnetic field was kept constant at $34 \mathrm{G}$, while the input power 


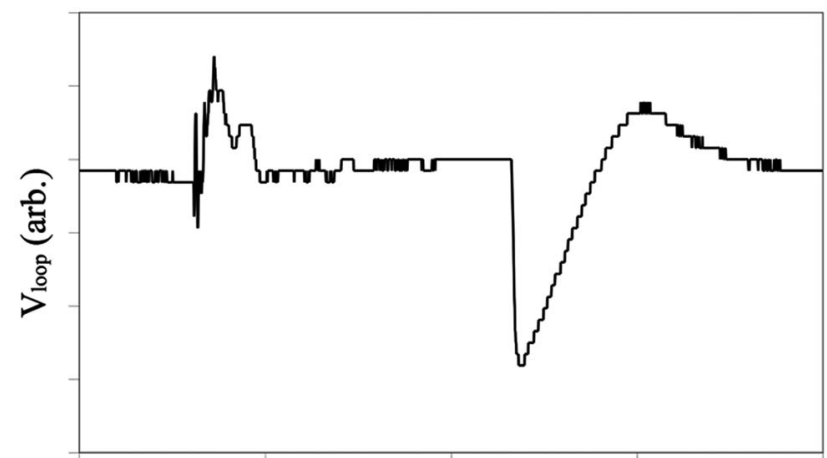

(a)

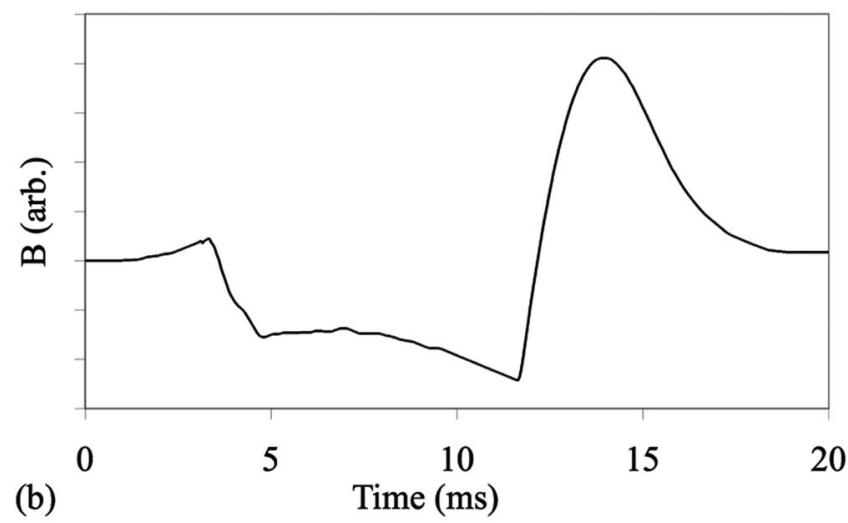

FIG. 6. Temporal evolution of the magnetic field by the plasma pressure. (a) Diamagnetic loop signal measured on the axis of the blue core and (b) time integration of signal showing decrease in magnetic field.

and hence the electron pressure was varied. The diamagnetism was determined by the decrease in the magnetic field when the plasma is on. For the conditions of the present experiments, the largest value of diamagnetism was found to be $1.8 \%$. It was found that the diamagnetic nature of the plasma increased linearly with increasing plasma beta.

The value of beta for various magnetic fields is shown in Fig. 9(a). Beta decreases with increasing magnetic field although not linearly. Since the plasma density and electron temperature are only weakly dependent on the magnetic field for the operating parameters explored here, it is found that beta decreases as the inverse square of the magnetic field, as

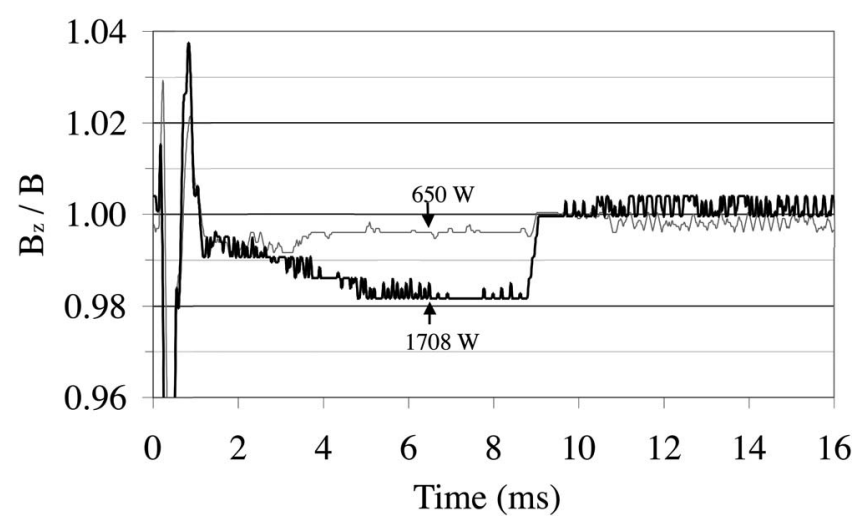

FIG. 7. Temporal evolution of the normalized magnetic field strength measured at the radial center by the Hall probe for two input powers.

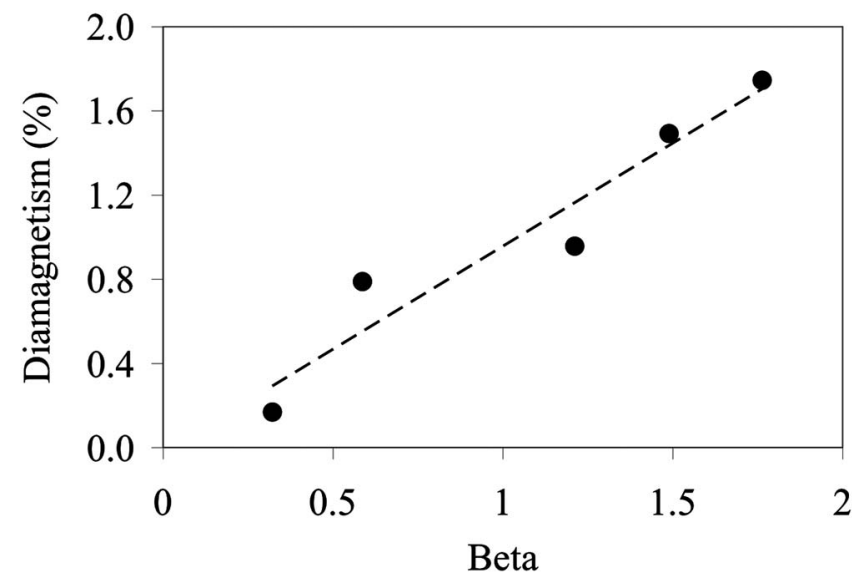

FIG. 8. The diamagnetism dependence on the plasma beta.

expected. Figure 9(b) shows the calculated Alfvén velocity $\left[V_{\mathrm{A}}=B /\left(4 \pi n M_{+}\right)^{1 / 2}\right]$ and ion acoustic velocity as a function of plasma beta for the experimentally measured densities and temperatures. The dashed line corresponds to an Alfvén velocity, calculated from $\beta=\left(u_{\mathrm{B}} / V_{\mathrm{A}}\right)^{2}$ for an electron temperature of $8 \mathrm{eV}$. When $\beta>1$, the Alfvén velocity becomes less than the ion acoustic velocity and according to general theory, the magnetized plasma becomes weakly selfmagnetized, with the external magnetic field no longer influencing the flowing plasma.
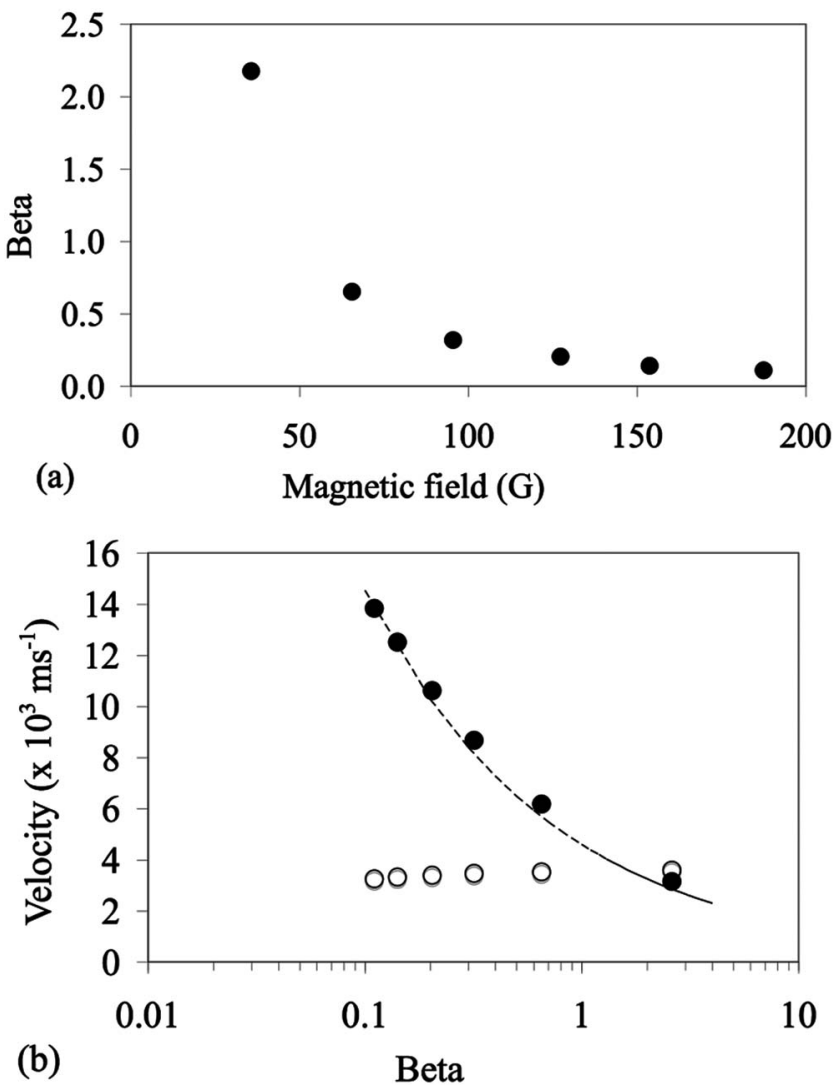

FIG. 9. (a) The dependence of beta on the applied magnetic field. (b) The measured Alfvén velocity (solid circles), ion acoustic velocity (open circles), and the calculated Alfvén velocity for a constant ion acoustic velocity of $4600 \mathrm{~ms}^{-1}\left(T_{e}=8 \mathrm{eV}\right)$. 


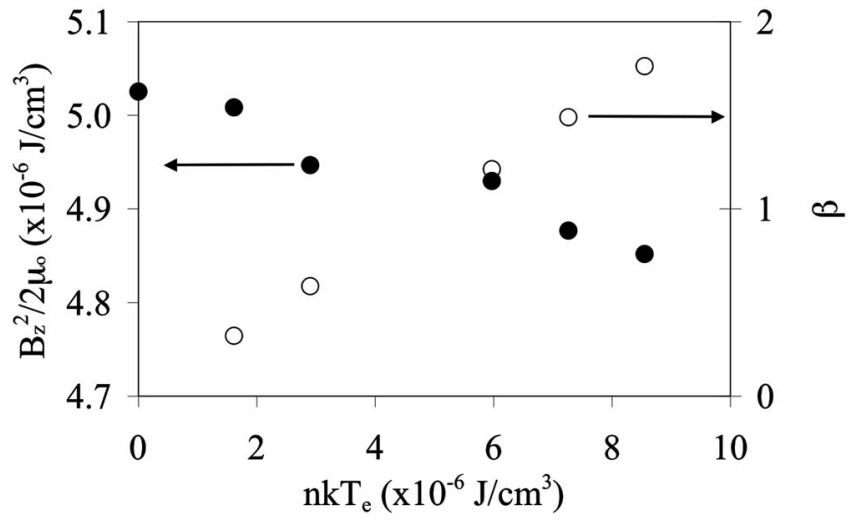

FIG. 10. Dependence of the magnetic pressure and plasma beta vs electron pressure.

The plasma diamagnetic properties are summarized in Fig. 10. For all operating conditions in our experimental investigation, we do not observe a complete expulsion of the magnetic field, even though $\beta>1$. Hence, the diamagnetic behavior cannot be described by the pressure balance equation: $B_{0}^{2} / 2 \mu_{0}=n k T_{e}+B_{z}^{2} / 2 \mu_{0}$. According to this equation, the magnetic field should be completely expelled for $\beta=1$. Previously, this deviation from the predictions has been suggested to be due to the electron magnetohydrodynamics characteristics of the plasma due to low values of the magnetic field that leaves electrons magnetized but not ions. ${ }^{10}$ In our situation, the ions are magnetized, and the magnetic field diffusion time is so fast that it is essentially in the plasma at all times.

\section{B. Radial diamagnetic profile}

As discussed in Sec. III, the plasma kinetic pressure changes across the radius of the discharge. Hence, one would expect the magnetic field pressure to also change. Figure 11 shows the change in the magnetic field across the plasma radius for the two magnetic field cases. Also shown in Fig. 11 is the measured plasma kinetic pressure (lines) corresponding to each case (multiplied by a fit factor). The change in the magnetic field measured by the Hall probe is verified by the change in the plasma pressure measured by a Lang-

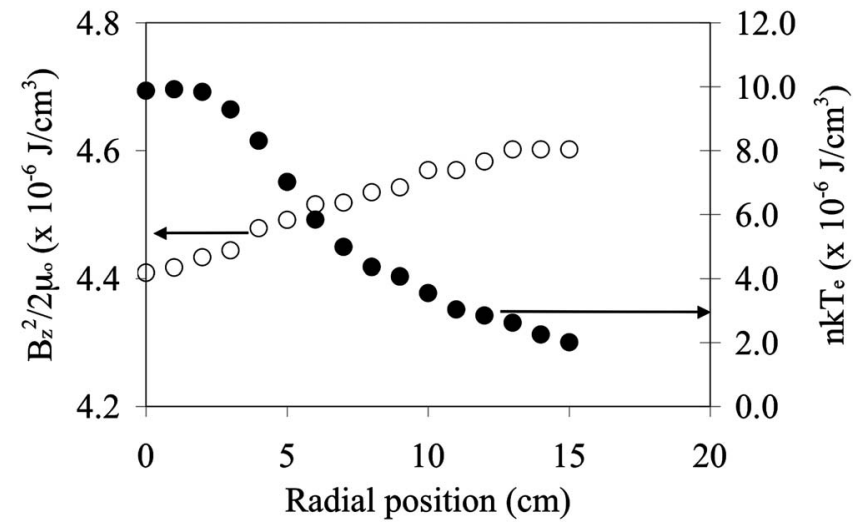

FIG. 12. The radial profile of the magnetic field pressure and electron pressure for $34 \mathrm{G}$.

muir probe. It is observed that the magnetic field displays the strongest decrease at the plasma center, which corresponds to the maximum in the plasma pressure. As the Hall probe is moved radially outwards from the center, the change in the magnetic field decreases and returns to the applied external magnetic field value, corresponding to a decrease in the plasma pressure.

The measured values for the radial variation of the electron pressure $\left(n k T_{e}\right)$ and the magnetic field pressure for the $34 \mathrm{G}$ case are shown in Fig. 12. The plasma pressure is a maximum where the magnetic field pressure is a minimum, and vice versa. The radial change in the plasma beta according to these measurements is shown in Fig. 13 for the two magnetic field cases. For a magnetic field of $34 \mathrm{G}$, beta is $\sim 2.2$ in the center and decreases to $\sim 0.5$ at $r=15 \mathrm{~cm}$. For a magnetic field of $185 \mathrm{G}$ the value for beta is much lower, being $\sim 0.15$ in the center and less than 0.02 at $r=7 \mathrm{~cm}$. Furthermore, the FWHM decreases with increasing magnetic field. The plasma observed in the diffusion chamber can therefore be described as a high-beta plasma in the blue core surrounded by a low-beta plasma.

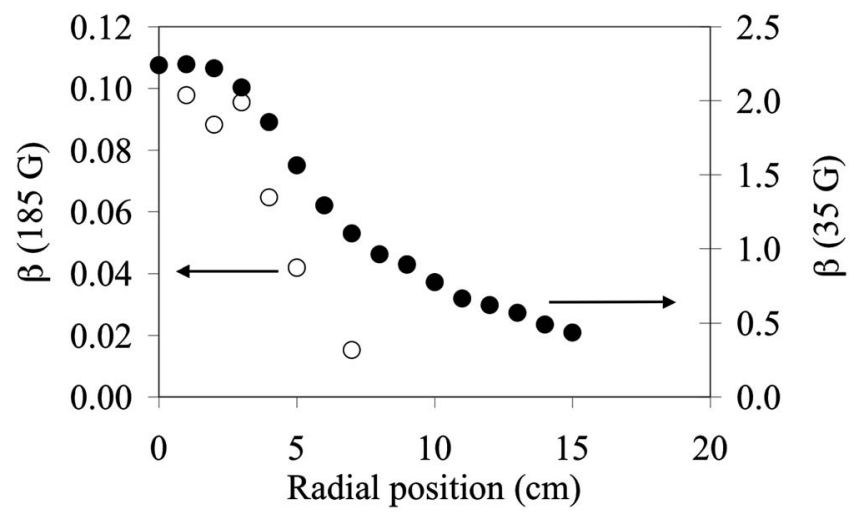

FIG. 13. The radial profile of beta determined from the measured electron density, electron temperature and magnetic field.

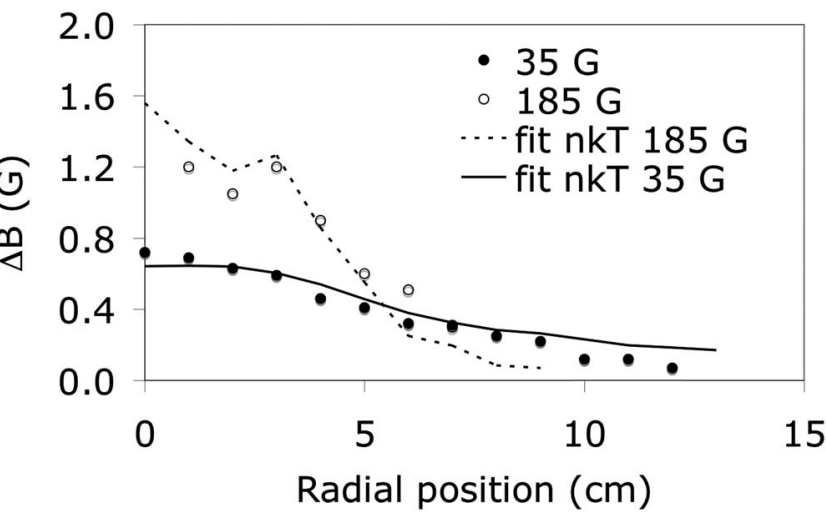

FIG. 11. The radial variation of the change in magnetic field strength by the 


\section{CONCLUSIONS}

The properties of a high-density magnetically confined plasma have been investigated experimentally. When the discharge is operated at high power and for magnetic fields greater than $34 \mathrm{G}$, a column of blue light is observed in the diffusion chamber. This blue core is a highly ionized plasma with a FWHM of $\sim 10-15 \mathrm{~cm}$. When using a magnetic field of $34 \mathrm{G}$, the magnetic field is found to reduce by $2 \%$ at the discharge center. The plasma diamagnetism decreases for decreasing plasma electron pressure and also for increasing magnetic fields. Although the plasma beta can be greater than 1, only a weak diamagnetism is ever observed for our discharge conditions. The decrease in the magnetic field is spatially dependent. Outside of the blue core a low-beta plasma exists and the magnetic field is only weakly, if at all, affected by the plasma. Hence, the plasma in the diffusion chamber is separated into a high-beta plasma in the center and a low-beta plasma at the edge.

Our main conclusion for this work is that although the electron pressure can exceed the magnetic field pressure, the magnetic field penetrates the plasma in a time faster than the plasma rise time, so that only a slight perturbation of the magnetic field is ever observed.

${ }^{1}$ G. A. Gary, Sol. Phys. 203, 71 (2001).

${ }^{2}$ H. H. Hamabata, Astrophys. J. 406, 563 (1993).

${ }^{3}$ H. Karimabadi, D. Krauss-Varban, and N. Omidi, Phys. Plasmas 2, 4177 (1995).

${ }^{4}$ M. Ono, Phys. Plasmas 2, 4075 (1995).

${ }^{5}$ F. F. Chen, Introduction to Plasma Physics and Controlled Fusion (Plenum, New York, 1984), Vol. 1.

${ }^{6}$ A. Miura, S. Ohtani, and T. Tamao, J. Geophys. Res. 94, 15231 (1989).

${ }^{7}$ S. Ohtani, A. Miura, and T. Tamao, Planet. Space Sci. 37, 567 (1989).

${ }^{8}$ F. J. Wessel, R. Hong, J. Song, A. Fisher, N. Rostoker, A. Ron, R. Li, and R. Y. Fan, Phys. Fluids 31, 3778 (1988).

${ }^{9}$ R. Hong, F. J. Wessel, J. Song, A. Fisher, and N. Rostoker, J. Appl. Phys. 64, 73 (1988).

${ }^{10}$ R. L. Stenzel and J. M. Urrutia, Phys. Plasmas 7, 4450 (2000).

${ }^{11}$ R. W. Boswell, Phys. Lett. 33A, 457 (1970).

${ }^{12}$ R. W. Boswell, Plasma Phys. Controlled Fusion 26, 1147 (1984).

${ }^{13}$ R. W. Boswell and R. K. Porteous, Appl. Phys. Lett. 50, 1130 (1987).

${ }^{14}$ P. Zhu and R. W. Boswell, Phys. Rev. Lett. 63, 2805 (1989).

${ }^{15}$ A. W. Degeling, T. E. Sheridan, and R. W. Boswell, Phys. Plasmas 6, 1641 (1999). 